\title{
Accuracy of the SpineNav3DTM Ultrasound Technology in Estimating the Epidural Space Depth for Epidural and Spinal Insertion in Pregnant Obese Patients
}

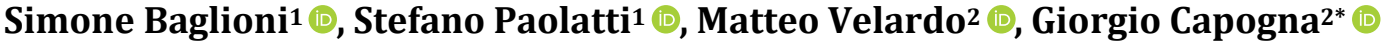 \\ ${ }^{1}$ Department of Anesthesia, ASST-S.Gerardo-Monza, Monza, Italy \\ ${ }^{2}$ European School of Obstetric Anesthesia, Roma, Italy \\ Email: *capogna.eesoa@gmail.com
}

How to cite this paper: Baglioni, S., Paolatti, S., Velardo, M. and Capogna, G. (2021) Accuracy of the SpineNav3DTM Ultrasound Technology in Estimating the Epidural Space Depth for Epidural and Spinal Insertion in Pregnant Obese Patients. Open Journal of Anesthesiology, 11, 221-228. https://doi.org/10.4236/ojanes.2021.118022

Received: June 25, 2021

Accepted: July 31, 2021

Published: August 3, 2021

Copyright $\odot 2021$ by author(s) and Scientific Research Publishing Inc. This work is licensed under the Creative Commons Attribution International License (CC BY 4.0).

http://creativecommons.org/licenses/by/4.0/

(c) (i) Open Access

\begin{abstract}
Introduction: Ultrasound facilitates neuraxial puncture in obese parturients. Unfortunately, the widespread adoption of neuraxial ultrasound may be limited by the lack of technical expertise or the limited availability of the equipment. A wireless portable ultrasound device (Accuro, Rivanna Medical, Charlottesville, VA) with automated pattern recognition software (SpineNav3DTM technology) has been introduced to obtain the automated real-time identification of interspaces and epidural depth. The primary objective of this study was to assess the accuracy of the SpineNav3DTM ultrasound technology (Accuro) in estimating the epidural space depth compared to the standard ultrasound examination in pregnant obese patients. The secondary aim was to compare the ultrasound measurements with the measured needle depth during epidural and spinal insertion. Methods: The study was conducted at S Gerardo Hospital, Monza, Italy from March 2021 to April 2021. Obese laboring women requesting epidural analgesia or undergoing elective cesarean delivery under spinal anesthesia were recruited. All the subjects had their lumbar area scanned for the measurements of the depth of the epidural space by the SpineNav3DTM ultrasound technology and by the standard US and then both compared with the needle insertion depth in a double-blind fashion. Results: Forty-eight women were enrolled in the study. There was agreement $( \pm 0.25 \mathrm{~cm}$ ) between the epidural depth (in $\mathrm{cm}$ ) measured with the Accuro, versus the standard ultrasound. There was a significant difference between the mean depth of epidural space s measured by Accuro or Standard US and needle insertion depth $(\mathrm{P}<0.001)$. Conclusions: The handheld ultrasound system with $3 \mathrm{D}$ spine navigation technology can automatically
\end{abstract}


identify and measure the epidural depth with the same accuracy as the standard ultrasounds in obese pregnant women.

\section{Keywords}

Ultrasound, Handheld Ultrasound, Epidural Space, Epidural Technique,

Obstetrics

\section{Introduction}

The utilization of pre-procedure ultrasound assessment before neuraxial block in obstetrics has been associated with reduced risk of failed epidural analgesia, less risk of traumatic placements, a decreased number of attempts for epidural placement, and the decreased incidence of accidental dural puncture [1] [2]. Many parturients gain a significant amount of weight during pregnancy, and hence, many patients satisfy the requirement for obesity with a BMI $>30 \mathrm{~kg} / \mathrm{m}^{2}$ [3], making the identification of bony landmarks by palpation difficult [4]. Ultrasound facilitates neuraxial puncture in obese parturients [5] [6], even if the presence of increased adipose tissue may make ultrasound visualization of the epidural space more challenging [7]. Unfortunately, the widespread adoption of neuraxial ultrasound may be limited by the lack of technical expertise, difficulty in obtaining and interpreting the images, or the limited availability of the equipment.

A wireless portable ultrasound device (Accuro, Rivanna Medical, Charlottesville, VA) with automated pattern recognition software (SpineNav3DTM technology) programmed to calculate the depth to the epidural space and identify bony landmarks has been introduced in order to require less operator interpretation of ultrasound images while offering automated real-time identification of interspaces and epidural depth. This device has been reported to accurately determine the depth to the epidural space and to identify the correct interspace in normal-weight women receiving labor epidural analgesia [8] and to be capable of measuring the epidural depth with the same accuracy as the standard ultrasounds in normal weight term pregnant volunteers [9].

The primary objective of this study was to assess the accuracy of the SpineNav3DTM ultrasound technology (Accuro) in estimating the epidural space depth compared to the standard ultrasound examination in pregnant obese patients. The secondary aim was to compare the ultrasound measurements with the measured needle depth during epidural and spinal insertion.

\section{Methods}

The local ethics board approved this prospective, double-blinded study (Comitato Etico Università di Milano La Bicocca, ASST Monza, n.3269) which was registered at Clinical Trials.gov with the identifier NCT 04395573.

The study was conducted at S Gerardo Hospital, Monza, Italy from March 
2021 to April 2021.

Laboring women requesting epidural analgesia or women undergoing scheduled cesarean delivery under spinal anesthesia were recruited. Written informed consent was obtained from all enrolled patients. The inclusion criteria included a current BMI of $\geq 30 \mathrm{~kg} / \mathrm{m}^{2}$, American Society of Anesthesiologists physical class $\leq$ III, ages 18 - 45 years, term gestational age ( $\geq 37$ weeks), singleton pregnancy, and a request for an epidural for labor analgesia or neuraxial anesthesia for elective cesarean section. Women were excluded if there was a contraindication to neuraxial analgesia (such as bleeding diathesis, local anesthetic allergy), severe scoliosis, or previous spine surgery.

Obesity was classified according to the World Health Organization (WHO) categories (class I = BMI $30-34.9 \mathrm{~kg} / \mathrm{m}^{2}$, class II = BMI $35-39.9 \mathrm{~kg} / \mathrm{m}^{2}$, and class III $=$ BMI $\geq 40 \mathrm{~kg} / \mathrm{m}^{2}$ ). We planned to recruit 45 consecutive obese patients.

Women were placed in a sitting position and instructed to flex their lumbar spine.

All the subjects had their lumbar area scanned for the measurements of the depth of the epidural space (recorded in $\mathrm{cm}$ ) defined as the distance from the skin to the posterior complex (ligamentum flavum, epidural space, and posterior dura) by an anesthesiologist expert in ultrasound assessment. In order to identify the intervertebral levels, the investigator, using the standard ultrasound instrument (My Lab 25, ESAOTE, Genova-Italia) with a convex probe 2 - $5 \mathrm{MHz}$, moved the probe cephalad to obtain the classic hyperechoic saw-like image representing the articular processes, and counted the interspaces upward till the chosen interspace. The midline was identified to obtain the view of the complex formed by the ligamentum flavum dura mater to allow for the measurement of the depth of the epidural space. After having identified the interspace, the investigator marked it on the skin. The depth of the epidural space was measured both in the transverse median plane and the paramedian sagittal oblique plane [10].

The skin marker was used by another investigator who, being blinded to the previous measurements, measured the epidural depth by using the Accuro $^{\circ}$ (Rivanna Medical, LLC; Charlottesville, VA-USA) device with a $5 \mathrm{MHz}$ probe. In this way, both the investigators were blinded to the other's measurements.

The anesthesiologists performing the neuraxial techniques were blinded to the actual US measurements but were aware of the location of the optimal insertion point by using the marked interspace point.

All the neuraxial blocks were performed with a median approach. The Tuohy/ spinal needle was advanced in a horizontal plane at the interspace previously selected by the ultrasounds in all patients. Once the epidural space was entered in the epidural/subarachnoid space, the Tuohy/spinal needle was marked by the anesthesia provider with a sterile marker to indicate the insertion depth. For the purpose of the study Fletx Tip Plus ${ }^{\odot}$ Epidural Catheterization Set Arrow ${ }^{\odot} 17$ G 
(Arrow International, Inc. Subsidiary of Teleflex Incorporated 2400 Bonneville Road Reading, PA 19605 USA) and Temema ${ }^{\odot} 25$ G, 103 mm spinal needle (RM te me ma GmbH Mittelhöferstraße 34587 Felsberg-D) were used respectively for the epidural and spinal anesthesia.

\section{Statistical Analysis}

Descriptive statistics, including the mean and SD or median and interquartile range, were used for the quantitative variables, whereas absolute and relative frequency was used for the qualitative variables.

Assuming a Type I error rate of $5 \%(\alpha=0.05)$ and a Type II error rate of $20 \%$ $(\beta=0.2)$, a minimum of 45 subjects were required for this study to detect a maximum difference of $0.25 \mathrm{~cm}$ in the measurement of the distance between the skin and the epidural space between the two devices (standard ultrasound and Accuro).

Normality tests, Shapiro-Wilk was used to check if the variables were normally distributed. The Paired T-test was used to compare the standard ultrasound scanner measurements with those of the Accuro ultrasound scanner. The Bland-Altman plot was used to compare the depth of the epidural space obtained with the ultrasound measurement to those obtained by marking the needle.

The Bland-Altman analysis was performed to place the size of the differences between the two measurements in a more clinical context. In addition, we estimated the $95 \%$ limits of agreement (LOA) for the differences, which represents the differences likely to arise between the two measurements with a $95 \%$ probability. We fixed the limits of agreement between the epidural depth estimated by Accuro and standard US at $0.25 \mathrm{~cm}$.

\section{Results}

Forty-eight women were enrolled in the study, three women were withdrawn for failure to follow study protocol, and thus not analyzed for the primary outcome, leaving 45 for the analysis. Table 1 shows the demographics. All the patients were class I and II obese pregnant women.

Mean depth of epidural space $(\mathrm{cm})$ as measured by Accuro, Standard Us, or needle insertion is reported in Table 2. There was a significant difference between the mean depth of epidural space as measured by Accuro or Standard US and needle insertion depth and both ultrasound devices consistently estimated an epidural depth shallower than the needle measured depth $(\mathrm{P}<0.001)$.

A Bland Altman plot showing the level of the agreement between the epidural depth (in $\mathrm{cm}$ ) measured with the Accuro, versus the standard ultrasound is reported in Figure 1. Horizontal lines are drawn at the mean difference and at the limits of agreement, which are defined as the means difference plus and minus 1.96 times the standard deviation of the differences. The mean difference is very close to $0.25 \mathrm{~cm}$ which was our pre-determined clinical difference endpoint. 
Table 1. Demograhics.

\begin{tabular}{cccc}
\hline & Mean/N & SD & 95\% CI \\
\hline Age (yrs) & 32.67 & 5.04 & $31.1-34.24$ \\
Weight (kg) & 96.81 & 19.92 & $90.6-103.01$ \\
BMI & 35.96 & 6.03 & $34.08-37.83$ \\
Parity (Nulliparous/Multiparous) & $39 / 6$ & & \\
Intervention (C-Section/Labor analgesia) & $18 / 27$ & & \\
Anesthesia (Epidural/Spinal) & $32 / 13$ & & \\
\hline
\end{tabular}

Table 2. Mean depth of epidural space $(\mathrm{cm})$ as measured by Accuro, Standard Us or needle insertion.

\begin{tabular}{|c|c|c|c|}
\hline L2-3 & mean & SD & $95 \% \mathrm{CI}$ \\
\hline Accuro* & 5.74 & 1.19 & $(4.93-6.53)$ \\
\hline Standard US & 6.00 & 1.14 & $(5.22-6.76)$ \\
\hline Needle insertion depth ${ }^{* *}$ & 6.51 & 1.06 & $(5.79-7.22)$ \\
\hline L3-4 & mean & SD & $95 \% \mathrm{CI}$ \\
\hline Accuro* & 5.54 & 0.88 & $(5.22-5.84)$ \\
\hline Standard US & 5.81 & 0.84 & $(5.64-6.13)$ \\
\hline Needle insertion depth ${ }^{* *}$ & 6.25 & 0.92 & $(5.98-6.51)$ \\
\hline
\end{tabular}

${ }^{*} \mathrm{NS}$ vs Standard US; ${ }^{* *} \mathrm{P}<0.001$ vs Standard US and Accuro within the limit agreement of $\pm 0.25 \mathrm{~cm}$.

Bland Altman ( standard US vs Accuro)

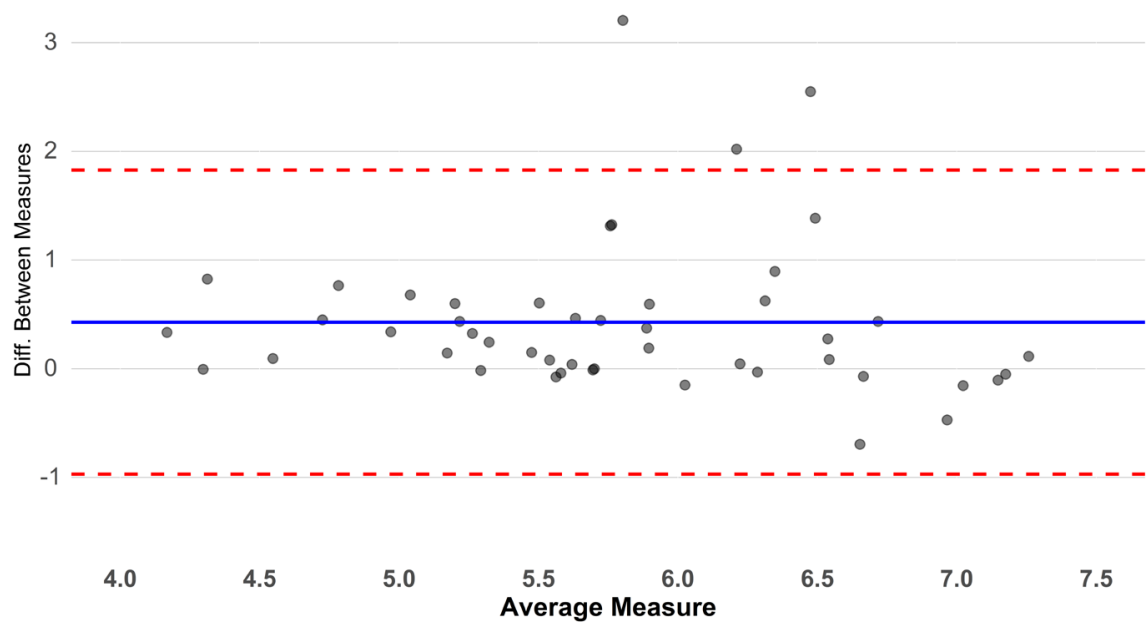

Figure 1. Bland Altman plot showing the level of the agreement between the epidural depth (in $\mathrm{cm}$ ) measured with the Accuro, versus the standard ultrasound. Horizontal lines are drawn at the mean difference and at the limits of agreement, which are defined as the means difference plus and minus 1.96 times the standard deviation of the differences. The mean difference is very close to $0.25 \mathrm{~cm}$ which was our pre-determined clinical difference endpoint.

\section{Discussion}

Obesity complicates the anatomical landmarks needed for epidural space locali- 
zation. The presence of adipose tissue together with the pregnancy-induced softening of the soft tissues and ligaments, may increase technical difficulties when identifying the epidural space by the loss-of-resistance technique.

Standard ultrasonography has been demonstrated to be a useful tool to predict the depth of the epidural space in obese parturients. In these patients, ultrasound can also reliably determine the skin puncture site [6].

However, widespread adoption of neuraxial ultrasound may be limited by technical expertise, difficulty in obtaining and interpreting the images, or limited storage space for ultra-sound equipment [1].

Previous studies have reported that the SpineNav3DTM ultrasound technology can accurately determine the depth to the epidural space and correctly identify the interspace in normal-weight women receiving labor epidural analgesia [11] and determine the epidural depth with the same accuracy as the standard ultrasounds in term pregnant women [9].

The major finding of our study is that the SpineNav3DTM ultrasound technology also provided comparable and accurate epidural depth estimates in class I and II obese pregnant women.

The SpineNav3DTM ultrasound technology compact portable device requires less operator interpretation of ultrasound images, while offering automated real-time identification of interspaces and epidural depth and this feature may be useful in many clinical settings where there is a lack of equipment or of a physician's adequate training or the need for rapid bedside use.

However, the Accuro device is designed to visualize bone landmarks and provides only an automatic epidural location and depth and therefore cannot be compared to and used as an ultrasound instrument but only as a "pointer". In addition, although the Accuro ultrasound system can gain ultrasound images in any orientation, the lumbar spine algorithm only assists with anatomical landmark recognition when imaging in the transverse plane, even if it has been reported that transverse plane imaging is adequate for recognizing the midline and the depth of the epidural space [10].

Interestingly, both ultrasound devices consistently estimated an epidural depth shallower than the needle measured depth with no observable differences between the two techniques. This well-known feature has been reported to usually be in the range of 1 to $13 \mathrm{~mm}$ [12] and may be due to the probe pressure exerted by the operator that compresses subcutaneous tissues which, in turn, decreases the depth to the epidural space as compared to the actual depth of clinical placement. The tendency of ultrasound to underestimate needle insertion introduces, however, a safety margin for clinicians.

\section{Limitation}

A limitation of the study might be that, although the anesthesiologists performing the neuraxial techniques were blinded to the actual US measurements, we did provide them with the location of the optimal insertion point. This might 
have introduced the potential for bias. However, we believe that it was ethically important to disclose this estimate based on the current knowledge of spinal US due to its beneficial contribution in potentially more difficult epidural procedures such as in the case of obese pregnant women.

\section{Summary}

In summary, the SpineNav3DTM ultrasound technology-based device appears to accurately determine the depth of the epidural space in moderately obese women receiving epidural labor epidural analgesia or spinal anesthesia for cesarean section.

\section{Conflicts of Interest}

The authors declare no conflicts of interest regarding the publication of this paper.

\section{References}

[1] Grau, T., Leipold, R.W., Conradi, R., Martin, E. and Motsch, J. (2002) Efficacy of Ultrasound Imaging in Obstetric Epidural Anesthesia. Journal of Clinical Anesthesia, 14, 169-175. https://doi.org/10.1016/S0952-8180(01)00378-6

[2] Schnabel, A., Schuster, F., Ermert, T., Eberhart, L.H., Metterlein, T. and Kranke, P. (2012) Ultrasound Guidance for Neuraxial Analgesia and Anesthesia in Obstetrics: A Quantitative Systematic Review. Ultraschall in der Medizin, 33, E132-E137. https://doi.org/10.1055/s-0029-1245724

[3] Simmons, D. (2011) Diabetes and Obesity in Pregnancy. Best Practice \& Research, Clinical Obstetrics \& Gynaecology, 25, 25-36. https://doi.org/10.1016/j.bpobgyn.2010.10.006

[4] Kula, A.O., Riess, M.L. and Ellinas, E.H. (2017) Increasing Body Mass Index Predicts Increasing Difficulty, Failure Rate, and Time to Discovery of Failure of Epidural Anesthesia in Laboring Patients. Journal of Clinical Anesthesia, 37, 154-158. https://doi.org/10.1016/j.jclinane.2016.11.010

[5] Wang, Q., Yin, C. and Wang, T.L. (2012) Ultrasound Facilitates Identification of Combined Spinal-Epidural Puncture in Obese Parturients. Chinese Medical Journal, 125, 3840-3843.

[6] Balki, M., Lee, Y., Halpern, S. and Carvalho, J.C. (2009) Ultrasound Imaging of the Lumbar Spine in the Transverse Plane: The Correlation between Estimated and Actual Depth to the Epidural Space in Obese Parturients. Anesthesia and Analgesia, 108, 1876-1881. https://doi.org/10.1213/ane.0b013e3181a323f6

[7] Grau, T., Leipold, R.W., Horter, J., Conradi, R., Martin, E. and Motsch, J. (2001) The Lumbar Epidural Space in Pregnancy: Visualization by Ultrasonography. British Journal of Anaesthesia, 86, 798-804. https://doi.org/10.1093/bja/86.6.798

[8] Seligman, K.M., Weiniger, C.F. and Carvalho, B. (2018) The Accuracy of a Handheld Ultrasound Device for Neuraxial Depth and Landmark Assessment: A Prospective Cohort Trial. Anesthesia and Analgesia, 126, 1995-1998. https://doi.org/10.1213/ANE.0000000000002407

[9] Capogna, G., Baglioni, S., Milazzo, V. and Vitale, A. (2018) Accuracy of the SpineNav3DTM Technology to Measure the Depth of Epidural Space: A Comparison 
with the Standard Ultrasound Technique in Pregnant Volunteers. Open Journal of Anesthesiology, 8, 113-122. https://doi.org/10.4236/ojanes.2018.84012

[10] Sahota, J.S., Carvalho, J.C., Balki, M., Fanning, N. and Arzola, C. (2013) Ultrasound Estimates for Midline Epidural Punctures in the Obese Parturient: Paramedian Sagittal Oblique Is Comparable to Transverse Median Plane. Anesthesia and Analgesia, 116, 829-835. https://doi.org/10.1213/ANE.0b013e31827f55f0

[11] Carvalho, B., Seligman, K.M. and Weiniger, C.F. (2019) The Comparative Accuracy of a Handheld and Console Ultrasound Device for Neuraxial Depth and Landmark Assessment. International Journal of Obstetric Anesthesia, 39, 68-73. https://doi.org/10.1016/j.ijoa.2019.01.004

[12] Perlas, A., Chaparro, L.E. and Chin, K.J. (2016) Lumbar Neuraxial Ultrasound for Spinal and Epidural Anesthesia: A Systematic Review and Meta-Analysis. Regional Anesthesia and Pain Medicine, 41, 251-260.

https://doi.org/10.1097/AAP.0000000000000184 